Perhaps the methods used to control reentrant waves in excitable media ${ }^{10}$ may also be applied to control fibrillation in human patients.

Arun V. Holden is at the Centre for Nonlinear

Studies, University of Leeds, Leeds LS2 9JT, UK.

\section{e-mail:arun@cbiol.leeds.ac.uk}

1. Gray, R. A., Pertsov, A. M. \& Jalife, J. Nature 392, 75-78 (1998)

2. Witkowski, F. X. et al. Nature 392, 78-82 (1998).

3. http://www.nature.com

4. Clayton, R. H., Murray, A. \& Campbell, R. W. F. J. Cardiovasc. Electrophysiol. 6, 616-624 (1995).

5. Holden, A. V. Nature 387, 655-666 (1997).
6. Biktashev, V. N. \& Holden, A. V. Chaos 8, 48-56 (1998)

7. Keener, J. \& Panfilov, A. V. in Cardiac Electrophysiology - From Cell to Bedside 2nd edn (eds Zipes, D. \& Jalife, J.) 335-347 (Saunders, Philadelphia, 1995)

8. Garfinkel, A. et al. J. Clin. Invest. 99, 305-314 (1997).

9. Pumir, A. \& Krinsky, V. I. Physica-D 91, 205-219 (1996).

10. Biktashev, V. N. \& Holden, A. V. Chaos Solitons Fractals 5, 575-622 (1995)

11. Winfree, A. T., Caudle, S., Chen, G., McGuire, P. \& Szilagyi, Z Chaos 6, 617-626 (1996).

12. Biktashev, V. N. in Computational Biology of the Heart (eds Panfilov, A. V. \& Holden, A. V.) 137-171 (Wiley, Chichester, 1997).

13. Nielsen, P. M. F., Le Grice, L. J., Smaill, B. H. \& Hunter, P. J. Am. J. Physiol. 260, H1365-H1378 (1991).

\title{
Lasers blow a bigger bubble
}

\section{Detlef Lohse}

S norkellers enjoy listening to drops falling on the surface of the sea. Most of the sound is not generated when the drops hit the surface, but when the little voids in the water that form after the collision collapse ${ }^{1}$. Kinetic energy is transferred into sound, as well as into thermal energy. But what about light? The typical kinetic energy of a falling molecule in a drop is only $10^{-6} \mathrm{eV}$, whereas the typical energy of a photon of light is in the $\mathrm{eV}$ range, so we would need an energy concentration of six orders of magnitude to produce light.

The voids formed by falling raindrops may not collapse violently enough to emit light, but Ohl, Lindau and Lauterborn ${ }^{2}$ of the University of Göttingen were able to detect light on collapse of an artificially created void. By firing an eight-nanosecond infrared laser pulse (containing just $20 \mathrm{~mJ}$ of energy) into water, they can cause a single bubble to nucleate at the laser focus. The bubble fills with dissolved gas and water vapour and grows up to three millimetres in diameter with a volume about 100-1,000 times larger than its equilibrium value. As the pressure inside the bubble is much less than that outside, the ambient pressure makes the bubble implode. At collapse, the authors detect up to $3 \times 10^{7}$ photons.

The authors call this phenomenon single-cavitation bubble luminescence (SCBL). It had already been observed in the 1970 s by Russian groups ${ }^{3}$, but no quantitative measurements of the light emission could then be made. SCBL resembles singlebubble sonoluminescence (SBSL), a peculiar phenomenon discovered $^{4,5}$ in 1990 . In SBSL a single bubble is trapped in an oscillating sound field and repeatedly collapses and emits light ${ }^{4-6}$.

Neither in SCBL nor in SBSL are the details of the light production process understood. What is already clear in both phenomena is that, on collapse, the gas inside the bubble is compressed so that it heats up, as in a pumped-up bicycle tyre. Here, however, it is heated rapidly to a temperature of at least sev- eral thousand kelvin, so visible light can be emitted. The three most likely light-emission mechanisms are ordinary thermal blackbody radiation, collision-induced emission, and bremsstrahlung.

The new method may allow measurements that shed some light on the emission mechanism, as the created bubbles are up to 1,000 times larger in volume than those of SBSL. SCBL also offers the opportunity to study bubble-bubble interactions, because two bubbles can be nucleated with controlled relative distance and phase. A better knowledge of bubble-bubble interactions will be the first step towards understanding multi-bubble sonoluminescence (MBSL), the light emission by thousands of bubbles in a bubble cloud under oscillating high pressure. Here, the bubbles disturb one another, so the collapses are much weaker on average, and the spectra of the emitted light have distinct lines ${ }^{7}$, in contrast ${ }^{6}$ to those of SBSL - implying that the gas in SBSL is so hot that emission lines are thermally smeared out. Ohl et al. ${ }^{3}$ have already taken a step towards studying bubble interactions by generating a bubble near a wall. As expected, the symmetry breaking due to the wall made the collapse less spherical, resulting in less light.

MBSL is of particular interest to chemists, as the kinetic energy of collapsing bubbles can be transformed not only into light but also into chemical reactions. In an experiment reported last year, Suslick et al..$^{8}$ measured reaction rates in a $200-\mathrm{m}-\mathrm{s}^{-1}$ water jet. This strongly turbulent flow creates tiny bubbles, which collapse, heating the trapped gases and the water vapour and so partly dissociating them. The radicals formed can recombine to oxidative species such as $\mathrm{OH}^{*}$ and $\mathrm{H}_{2} \mathrm{O}_{2}$, which oxidate dissolved iodide to iodine easily detectable, as it forms a blue complex with starch. Although it was already known that such reactions can occur in acoustical cavitation (sonochemistry ${ }^{9}$ ), it is remarkable that — provided some starch is dissolved extremely strong stirring of a 1-molar potassi- um iodide solution may turn it blue.

Suslick et al. also discovered a counterintuitive water-temperature dependence of the reaction rate: as the water temperature increases the reaction rate decreases. The reason, presumably, is that the water vapour pressure strongly increases with temperature, and on fast compression a water-vapourfilled bubble does not heat up as easily as a nitrogen bubble, or even an inert gas bubble.

The common feature of all the phenomena discussed so far - the sound of rain, SCBL, chemistry-inducing hydrodynamical cavitation, MBSL and SBSL (in which chemical reactions also occur ${ }^{10,11}$ ) - is that a bubble collapses, forming a geometric singularity at its centre where extreme physical conditions are achieved. The main questions now are, how extreme can these conditions be, and what physical processes occur under such conditions?

Great progress towards answering these questions has been made by Gompf, Eisenmenger and co-workers ${ }^{12}$. With time-correlated single-photon counting, they were able to measure the width of SBSL light pulses. Their measurements, which have now been confirmed in other labs, show that the light can last as long as 300 ps. Moreover, the length of the light pulse is approximately the same for all observable frequencies, constraining theoretical models of the light production mechanism. For example, if the emission is simply black-body radiation, the temperature must reach at least 70,000 K.

It may be worth trying to increase the intensity of the bubble collapse by combining the methods employed in SCBL and SBSL. One could nucleate a bubble using a strong laser pulse in the pressure antinode of an oscillating sound field, so that the increasing sound pressure would accelerate the bubble collapse. In this way the temperatures and pressures inside the bubble could be pushed even further into the region of extreme states of matter, and time-resolved spectroscopy of a single light pulse might become possible.

Detlef Lohse is in the Department of Physics,

University of Marburg, Renthof 6, D-35032

Marburg, Germany.

e-mail:lohse@stat.physik.uni-marburg.de

1. Prosperetti, A., Crum, L. A. \& Pumphrey, H. C. J. Geophys. Res. 94, 3255-3259 (1989).

2. Ohl, C. D., Lindau, O. \& Lauterborn, W. Phys. Rev. Lett. 80, 393-396 (1998).

3. Buzukov, A. A. \& Teslenko, V. S. JETP Lett. 14, 189-191 (1971).

4. Gaitan, D. F. Thesis, Univ. Mississippi (1990).

5. Crum, L. A. Phys. Today 47, 22-29 (1994).

6. Barber, B. P., Hiller, R. A., Löfstedt, R., Putterman, S. J. \& Weninger, K. R. Phys. Rep. 281, 65-144 (1997).

7. Matula, T. J., Roy, R. A., Mourad, P. D., McNamara, W. B. \& Suslick, K. S. Phys. Rev. Lett. 75, 2602-2605 (1995).

8. Suslick, K. S., Mdleleni, M. M. \& Ries, J. T. J. Am. Chem. Soc. 119, 9303-9304 (1997).

9. Suslick, K. S. Science 247, 1439-1445 (1990).

10. Lohse, D., Brenner, M. P., Dupont, T., Hilgenfeldt, S. \& Johnston, B. Phys. Rev. Lett. 78, 1359-1362 (1997).

11. Matula, T. J. \& Crum, L. A. Phys. Rev. Lett. 80, 865-868 (1998). 12. Gompf, B., Günther, R., Nick, G., Pecha, R. \& Eisenmenger, W Phys. Rev. Lett. 79, 1405-1408 (1997). 